\title{
Analysing the impact of traffic incidents on express road traffic flow using FREEVAL
}

\author{
Eliza Ciszewska-Kulwińska ${ }^{1}$, Aleksandra Romanowska $^{1, *}$, and Wojciech Kustra $^{1}$ \\ ${ }^{1}$ Gdansk University of Technology, Faculty of Civil and Environmental Engineering, Department of \\ Highway and Transportation Engineering, 11/12 Narutowicza Str., 80-233, Gdansk, Poland
}

\begin{abstract}
Traffic incidents occurring on motorways or express roads cause disruptions and deteriorate traffic conditions. The impact will differ depending on the type of incident, its duration and space blocked on the roadway and can be measured with e.g. average speed reduction, extension of travel time, time lost or overall costs of traffic disruptions. The aim of the paper is to analyse this impact, based on data from the Tri-City Ring Road (Poland). The analyses were conducted on a macroscopic level, with the use of a simulation software FREEVAL. The analysed road section was divided into homogeneous basic, merge and diverge segments. Particular traffic disturbances were introduced into individual segments, in order to represent a traffic accident or short-term road works leading to a blocked shoulder, closure of 1 lane or 2 lanes for the duration of 15 to 60 minutes. The total of 150 scenarios were analysed. The results of the analyses helped to assess how travel time and vehicle speed change depending on the location, type and duration of the traffic incident. It was found, for example, that in the case of right shoulder blockage, travel time will not change significantly (up to $3 \%$ ) while the closing of 1 of 2 or 2 of 3 lanes will cause significant travel time extension (by over 500\%).
\end{abstract}

\section{Introduction}

Although designed to be the safest and most reliable parts of the road network, motorways and express roads are not incident-free despite their high standards. Depending on the type of incident (accident, collision, road works), its location, duration and consequences (e.g., blocking a shoulder or one or more lanes), disruptions and deteriorated driving conditions are a likely consequence [1].

In 2016, 688 accidents occurred on Poland's express roads and motorways which represents $2 \%$ of all accidents and nearly $11 \%$ of accidents on national roads [2]. Considering the length of these roads (Poland has more 3,500 km of motorways and express roads), per each $100 \mathrm{~km}$ there were 9 accidents within a year. While road accident data are easily available, reports of other incidents such as collisions, vehicles stopped on the road or road works are not. Collected by a variety of organisations, the data are frequently incomplete, especially when it comes to random and short-term incidents. Whatever the type of incident, traffic flows are always affected leading to delays and growing costs of road traffic.

\footnotetext{
* Corresponding author: aleksandra.romanowska@pg.edu.pl
} 
This sets the context for addressing how incidents change driving conditions and the quality of driver experience depending on the type of incident, its location, outcomes and duration. Understanding this can help to anticipate the effects on e.g. average speed, travel time, delays and level of service when a specific incident occurs. This kind of information can be important in the context of real-time traffic management (e.g. detours suggested to drivers) or in road network planning (to ensure adequate road efficiency).

The objective of the paper is to analyse the effects of road incidents on traffic on motorways and express roads using the example of the S6 express road (Tri-City Ring Road). A section was selected located Gdańsk, between the Gdańsk Lotnisko and Gdańsk Karczemki interchanges. The analyses were carried out using FREEVAL, an HCM-based software for simulating and assessing traffic conditions over longer sections of roads.

\section{Background}

A road incident can be defined as a non-recurring or irregular event that causes a change in traffic operations (e.g. reduction in capacity, average speed, longer travel time) or an abnormal change in demand [3]. Incidents can be planned such as road works, special or unplanned and random such as crashes, PDO (property damage only) accidents and other incidents e.g. disabled vehicles, oil spills, etc. According to the literature, road incidents both planned and unplanned, cause some disturbance and deteriorate traffic conditions by e.g. reducing average speed and road capacity [4-6] and by affecting travel time reliability [7-9].

Road incidents lead to temporary bottlenecks, places where road capacity changes as a result of narrower road cross-sections (parts of shoulders or lanes becoming blocked by stationary or damaged vehicles). When a bottleneck forms, vehicle stream flows are disturbed leading to the formation of a kinematic shock wave. As it spreads over time and space, traffic density increases fast and average speed of traffic stream upstream of the incident location decreases. This will be the case when the volume of traffic towards the bottleneck is greater than the maximum volume that can pass across the cross-section when it is narrower. Bottleneck effects on traffic can be described by using e.g. hydrodynamic analogy $[10,11]$, Cell Transmission Model [12], the queueing theory [13] or simulation models.

How a traffic incident will affect traffic will depend on a number of factors such as road and traffic factors, road cross-section (the number and width of lanes, shoulder width), traffic volume, speed limit and incident-related factors such as: the type (planned, unplanned), duration (until normal traffic is restored) and the consequences (e.g. 1 lane blocked, shoulder blocked) [1]. The effects can be seen from a micro (change of behaviour of individual drivers) and macroscopic perspective (change of vehicle stream traffic parameters). Knoop [14] reports that as drivers approach the scene of an incident, they reduce speed and almost double the distance between vehicles compared to normal driving conditions. From a macroscopic perspective road incidents change capacity in particular and the change depending on the consequences of the incident, may range from several to more than $80 \%[3,4,6,15,16]$ (Bląd! Nie można odnaleźć źródła odwołania.).

Table 1. The effect of road incident on capacity.

\begin{tabular}{|l|c|c|c|c|}
\hline \multirow{2}{*}{ Research } & \multicolumn{4}{|c|}{ Consequences of road incident } \\
\cline { 2 - 5 } & $\begin{array}{c}\text { Shoulder } \\
\text { blocked }\end{array}$ & $\begin{array}{c}\text { 1 of 2 lanes } \\
\text { blocked }\end{array}$ & $\begin{array}{c}\text { 1 of 3 lanes } \\
\text { blocked }\end{array}$ & $\begin{array}{c}\mathbf{2} \text { of 3 lanes } \\
\text { blocked }\end{array}$ \\
\hline Goolsby (1970) & $33 \%$ & N/A & $50 \%$ & $79 \%$ \\
\hline Qin \& Smith (2001) & N/A & N/A & $63 \%$ & $77 \%$ \\
\hline PB Farradyne (2000) & $1-19 \%$ & $65 \%$ & $51 \%$ & $83 \%$ \\
\hline HCM (2016) & $7-19 \%$ & $65 \%$ & $51 \%$ & $83 \%$ \\
\hline Knoop et al. (2009) & $28 \%$ & N/A & $64 \%$ & $82 \%$ \\
\hline
\end{tabular}


The literature includes analyses of how incidents change travel time which, depending on the consequences of the incident, may increase by some forty or several hundred percent $[7,8,15]$. Building on sensitivity analysis Qin et al. [15] pointed out that a $2 \%$ capacity reduction may increase individual delay by $11 \%$ or more. In addition, we know from the literature that road incidents increase travel time variability and reduce travel time reliability $[7,8]$.

The effects of road incidents on traffic are difficult to study. Having to analyse multiple cases and random events is demanding and the lack of historical data makes incident studies even more difficult. This can be alleviated by simulation tools. Used by a number of researchers, simulation tools are very helpful with complementing missing data or analysing scenarios whenever real data are difficult to obtain. One such tool is FREEVAL (FREeway EVALuation) designed for macroscopic analyses and based on the US' HCM method [4] and Daganzo's Cell Transmission Model [12]. It helps to analyse traffic on sections of uninterrupted flow facilities, both in undersaturated and oversaturated traffic conditions. In the case of oversaturated traffic conditions, the model is based on hydrodynamic analogy and assumes linear behaviour of the stream speed if vehicle density is greater than 28 vehicles $/ \mathrm{km} /$ lane, i.e. for congested traffic.

One of FREEVAL's features is that it can analyse the consequences in time and space of traffic disruptions such as road works, an accident, collision or bad weather. Following from the analysis, we can compare the changes in average speed and travel time, kilometres travelled, delays or use of capacity. Disruptions in the model are represented by capacity reductions on a segment as a result of a narrower cross-section, e.g. when a lane is blocked by road works. By manoeuvring parameters such as DAF (Demand Adjustment Factor), CAF (Capacity Adjustment Factor), SAF - Free Flow Speed Adjustment Factor and Driver Population Adjustment Factor, the model can be calibrated to achieve the best possible approximation to real conditions and real impacts of road incidents on traffic.

\section{Methodology}

The analyses presented in the paper were made for a section of the S6 express road within the Tri-City Ring Road located in Gdansk. This is a dual carriageway with 2 to 3 lanes in each direction. The Tri-City Ring Road is of particular importance for both transit, agglomeration, tourist and leisure traffic. The road's annual average daily traffic (AADT) exceeds 77,000 vehicles daily, and 100,000 vehicles per day in the summer months. High volumes cause the road to function at capacity, which is evident in particular at peak hours on weekdays and during the summer. Because this is such a busy road, collisions, accidents and other incidents are quite frequent. Between 2011-2015 there were 185 accidents involving 221 injuries and 15 fatalities. There were about 200 road works of which the majority caused the cross-section to decrease by 3 to $7 \mathrm{~m}$. While collisions and other incidents occur almost every day, the services register only some of them.

The analysis looks at a $3 \mathrm{~km}$ section between the interchanges of Gdańsk Lotnisko and Gdańsk Karczemki and features the Tri-City Ring Road's highest traffic volumes. The section consists of basic segments between the interchanges with varying cross-sections $(2 \times 2$, $2 \times 3$ ) and merge and diverge segments connected to the collector road where weaving maneuvers take place.

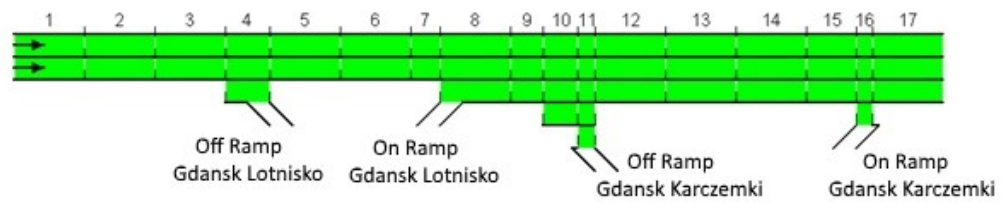

Fig. 1. Model of the analysed section in FREEVAL. 
A model was developed to represent the section using FREEVAL (fig. 1) with road and traffic parameters (i.e. road cross-section, share of heavy vehicles) to match the actual conditions. Traffic data were collected on a typical weekday in December 2014. The volumes on the main roadway in that time ranged from 2,100 to $3,400 \mathrm{~V} / \mathrm{h}$. The analyses concentrated on the afternoon peak (3-6 pm) consisting of twelve 15 minute intervals. Because the road services the conurbation the Driver Population Adjustment Factor was agreed to be 1. CAF and SAF were not calibrated due to a lack of data. To simplify the analysis, it was assumed that incidents do not change demand $(\mathrm{DAF}=1)$.

A total of 156 scenarios were built: baseline scenario (normal traffic, no incidents) and incident scenarios of varying duration (15-60 minutes), location and consequences (Table 2). The incidents simulated: a blocked shoulder, blockage of one of 2 and 3 lanes, blockage of 2 lanes (on three lane sections). Disruption in each scenario emerged in the same time interval, i.e. 15:15-15:30. For each scenario, in result of simulation, segment travel time was estimated, allowing to compare the effect of road incident depending on its location, duration and consequences.

Table 2. List of analysed scenarios.

\begin{tabular}{|l|c|c|c|}
\hline \multicolumn{3}{|c|}{ Road incident scenarios } & Scenarios - \\
total
\end{tabular}

In result of conducted simulations segment travel time was estimated for each scenario.

\section{Results}

The main focus of the analysis was to determine how travel time changed as a result of a road incident. The data were aggregated in time and space, i.e. each time interval and each segment was averaged over the entire period of analysis.

Travel time on the entire section in the no-disruptions scenario varies from interval to interval and is within 3.43-3.51 min. Given the traffic volume, travel time is slightly longer compared to free flow even if no external disruptions are added $(3.41 \mathrm{~min})$. While average speed is close to free flow speed, it varies between two and three lane sections (the difference being app. $5 \%$ ).

The scenarios were analysed and it was clear that road incidents change the conditions of traffic. However, depending on the incident's duration and consequences the effects will vary significantly, e.g. a blocked shoulder will affect traffic to a much lower degree than the blockage of half of the roadway. All of the scenarios were analysed and the results are shown in figures 2-4.

An observation was made that a blocked shoulder on a two lane segment does not have much of an effect on travel time. A blocked shoulder will usually affect traffic only while the road incident is in progress (fig. 2). In another intervals the travel times are similar and the lines in figure overlap. As we know from analyses, a closed shoulder increases travel time by a maximum of $3 \%$ on the entire section. Segments with three lanes are not really affected when a shoulder is blocked. 

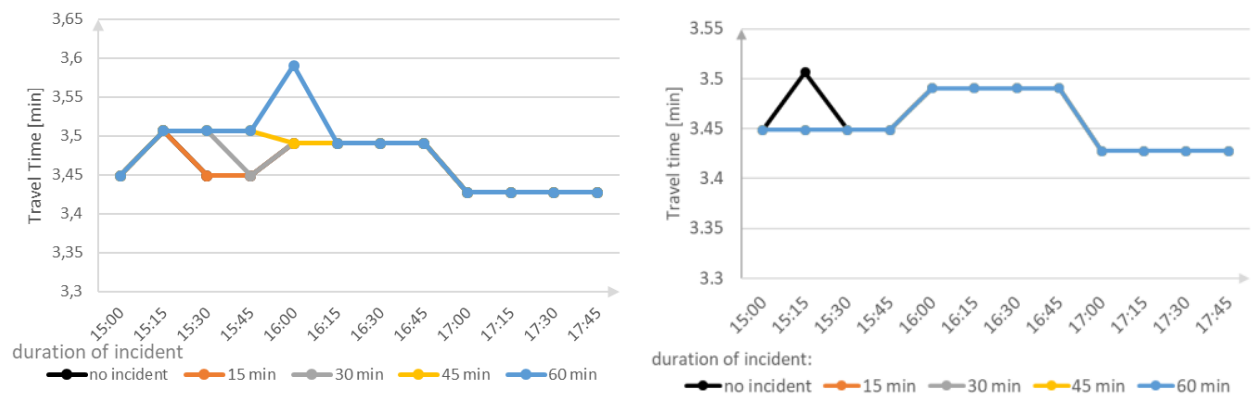

Fig. 2. Change in travel time as a result of a road incident blocking a shoulder on a segment with (a) two lanes - segment 3, (b) three lanes - segment 12.

When one lane is blocked, travel time increases on the analysed section on average by 1.5 times to slightly more than 2 times depending on the volume. The relationship between a longer travel time and traffic volume when one lane is blocked is close to a linear relationship. The worst of the 15 minute intervals in all of the scenarios on a two lane cross-section increases travel time by as much as $560 \%$. The longest time to restore normal traffic conditions to what they were before the incident is within 30 minutes after the incident dissipates. As you can see in figure 3 which shows examples of scenarios when 1 of 2 lanes and 1 of 3 lanes is blocked, the effects of the 1 of 2 lanes blockage are much worse than when 1 of 3 lanes are blocked with travel time increasing by up to $300 \%$.
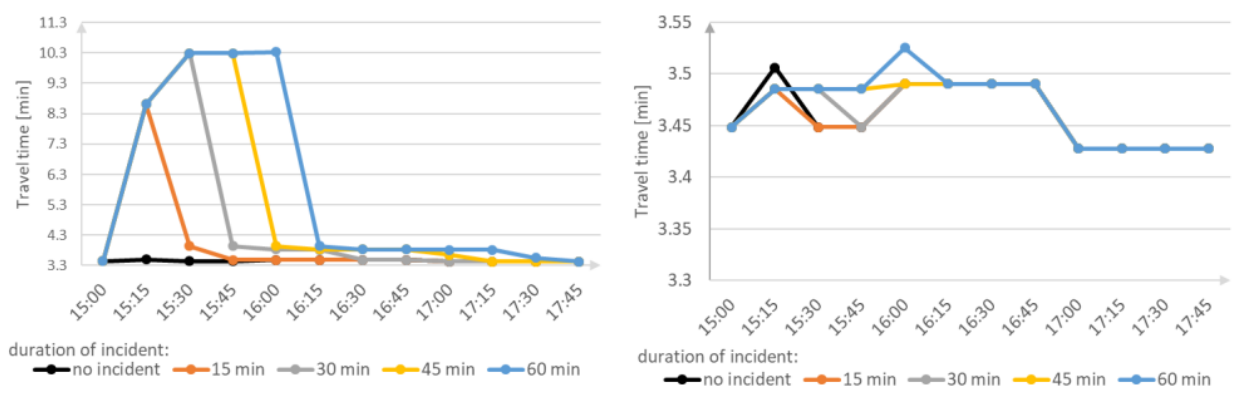

Fig. 3. Change in travel time as a result of a road incident blocking 1 lane on a segment with (a) two lanes - segment 3, (b) three lanes - segment 12.

Disruption to traffic is the biggest when the incident causes a blockage of 2 lanes on a 3 lane segment. When 2 lanes are blocked, travel time may increase by up to 3,600\%. As an example, if 2 lanes are blocked on segment 12 for 30 minutes, travel time compared to scenario 0 increased significantly and ran from 15:15 (start of incident) to 15:45. The delay continued for another 15 minutes after the incident ended and disappeared entirely only in the interval beginning at 16:45 (fig. 4). Travel time was the longest in the interval beginning at 15:30, which is 15 minutes after the incident. Travel time in that case was longer by $550 \%$ compared to a no-incident situation. Average speed while the incident lasted in the worst period dropped to $22 \mathrm{~km} / \mathrm{h}$. It was observed that in the second and third time interval the demand exceeded capacity, further causing a blockage. It was also observed that if 2 lanes are blocked on a 3 lane cross-section, travel time increases even three times compared to when 1 lane is blocked on a 2 lane cross-section. 


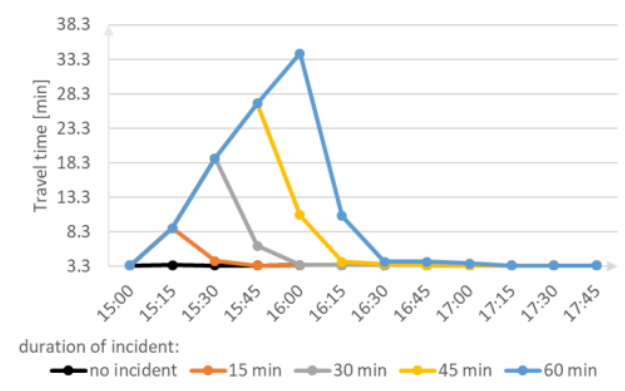

Fig. 4. Change in travel time as a result of a road incident blocking 2 lanes on a three lane segment (segment 12).

\section{Discussion and conclusion}

Many researchers have studied the effects of road incidents on traffic conditions. While they have demonstrated that both planned and random events reduce capacity and average speed leading to longer than expected travel times, the changes come as a result of a number of factors related to road and traffic conditions and the incident itself - the type, duration and consequences. With a multitude of possible options and randomness of events, empirical studies of road incidents and their effects on traffic are difficult and require frequent support from simulation tools.

The objective of the paper was to identify how road incidents change traffic conditions on motorways and express roads, using FREEVAL's simulation model, based on the US' method for highway capacity analysis. The conditions were measured using travel time and its length compared to the baseline scenario which was not affected by traffic disruptions. It was observed that a blocked shoulder will only affect traffic while the incident is in progress. When the blockage is over, traffic is restored to the condition from before the incident. Travel time increases slightly during shoulder blockage. When a blockage occurs on a 2 lane crosssection, delays are only in the range of several percent and are almost unnoticeable when a 3 lane cross-section is blocked. When 1 of 2 lanes are blocked the time for the consequences to dissipate is on average twice as long as the duration of the incident alone. This means that a blockage of 1 lane for 15 minutes affects traffic within 30 minutes from the incident, i.e. an extra 15 minutes after the incidents ends. In the worst case scenarios travel time increased (when incident duration was 60 minutes) by as much as 550\%. A blockage of 2 of 3 lanes on average increased the impact of the incident threefold compared to its duration, and so for a 15 minute incident its consequences continued for 45 minutes after it had started. In the worst case scenarios travel time increased by as much as 3,600\% when 2 of 3 lanes were blocked.

The use of the simulation model is somewhat limited because it cannot be easily calibrated to the conditions in Poland to understand the effects of road incidents (CAF and $\mathrm{SAF}$ ). Poland does not have relevant empirical research of this scale. On the one hand, information about disruptions is incomplete and may come from a number of sources. On the other hand, access to historical traffic data is difficult (a small number of continuous traffic count stations, lack of stationary devices to measure traffic flow parameters on road sections). This is why simulation tools can offer the necessary support for research. If, however, the model is not calibrated for the conditions in Poland, the results from the analysis may be inaccurate. As a consequence, further work should be designed to verify the results of modelling and draw conclusions about how strongly road incidents change traffic conditions on sections of motorways and express roads. 
The work presented in the paper was conducted under the RID-2B project "Modern methods for calculating capacity and evaluating traffic conditions on roads outside conurbations, including fast traffic roads" (OT2-2B/PK-PW-PG), delivered between 2016-2019 under Joint Initiative of the National Centre for Research and Development and the General Directorate for National Roads and Motorways "Development of Road Innovations".

\section{References}

1. H. Chen, Y. You, J. Zhou, L. Wang, Z. Shang, A Simplified Approach to Estimate the Urban Expressway Capacity after Traffic Accidents Using a Micro-Simulation Model, Adv. Mech. Eng. 5 pp. 656345 (2013). doi:10.1155/2013/656345

2. Polish National Road Safety Council, Road Safety Annual Report 2016 (2017)

3. PB Farradyne, Traffic Incident Management Handbook (Federal Highway Administration, 2000)

4. Transportation Research Board, Highway Capacity Manual 6th Edition: A Guide for Multimodal Mobility Analysis (Transportation Research Board of the National Academies, Washington, 2016)

5. V. Knoop, S. Hoogendoorn, H. van Zuylen, Capacity Reduction at Incidents: Empirical Data Collected from a Helicopter, Transp. Res. Rec. J. Transp. Res. Board. 2071 pp. 19-25 (2008). doi:10.3141/2071-03

6. V. Knoop, S. Hoogendoorn, K. Adams, Capacity Reductions at Incidents Sites on Motorways, Eur. J. Transp. Infrastruct. Res. 9 pp. 363-379 (2009)

7. B. Wright, Y. Zou, Y. Wang, Impact of Traffic Incidents on Reliability of Freeway Travel Times, Transp. Res. Rec. J. Transp. Res. Board. 2484 pp. 90-98 (2015). doi:10.3141/2484-10

8. H. Tu, H. van Lint, H. van Zuylen, The Effects of Traffic Accidents on Travel Time Reliability, pp. pp 79-84 (2008). doi:10.1109/ITSC.2008.4732581

9. P. Olszewski, T. Dybicz, K. Jamroz, W. Kustra, A. Romanowska, Assessing Highway Travel Time Reliability using Probe Vehicle Data, Transp. Res. Rec. J. Transp. Res. Board. pp. 036119811879671 (2018). doi:10.1177/0361198118796716

10. M.J. Lighthill, G.B. Whitham, On Kinematic Waves. II. A Theory of Traffic Flow on Long Crowded Roads, Proc. R. Soc. A Math. Phys. Eng. Sci. 229 pp. 317-345 (1955). doi:10.1098/rspa.1955.0089

11. G.F. Newell, A simplified theory of kinematic waves in highway traffic, Part I: General theory, Transp. Res. Part B Methodol. 27 pp. 281-287 (1993)

12. C.F. Daganzo, The Cell Transmission Model, part II: Network Traffic, Transp. Res. Part B Methodol. 29 pp. 79-93 (1995). doi:https://doi.org/10.1016/01912615(94)00022-R

13. I. Adan, J. Resing, Queueing Systems (Eindhoven University of Technology, 2015)

14. V.L. Knoop, Road Incidents and Network Dynamics Effects on driving behaviour and traffic congestion, Delft University of Techology, 2009

15. L. Qin, B.L. Smith, Characterization of Accident Capacity Reduction. Smart Travel Lab Report No. STL-2001-02 (UVA Center for Transportation Studies, 2001)

16. J. Zeeger, J. Bonneson, R. Dowling, P. Ryus, M. Vandehey, W. Kittleson, N. Rouphail, B. Schroeder, A. Hajbabaie, B. Aghdashi, T. Chase, S. Sajjadi, R. Margiotta, L. Elefteriadou, Incorporating Travel Time Reliability into the Highway Capacity Manual. REPORT S2-L08-RW-1, 2014 